\title{
NON-TREATMENT OF PATENT DUCTUS ARTERIOSUS AND SHORT TERM OUTCOMES IN EXTREMELY LOW BIRTH WEIGHT INFANTS (ELBW)
}

\author{
S. Sathiyamurthy ${ }^{1}$, M. Thomson ${ }^{2}$, L. Tyszczuk ${ }^{2}$, A. Groves ${ }^{2}$ \\ ${ }^{I}$ Neonatal Unit, Chelsea \& Westminster Hospital, ${ }^{2}$ Neonatal Unit, Queen Charlotte's and Chelsea Hospital, \\ Imperial College Healthcare NHS Trust, London, UK
}

Background and aims: Optimal management of Patent Ductus Arteriosus(PDA) in ELBW infants is controversial and trials of therapeutic intervention have not shown benefit. In our unit we practice early extubation to CPAP, permissive hypercapnia and only consider PDA treatment when infants remain ventilator-dependent. The aim of this study is to audit PDA treatment in our unit and following discharge, and to compare short-term outcomes to published benchmarks.

Methods: Retrospective study of all ELBW infants who were inborn or admitted to our unit within 72 hours of birth from 2007-2009. All data were collected from comprehensive discharge summaries, validated against SEND and VON databases. Outcomes were compared with Vermont Oxford Network data ${ }^{1}(2004)$ by Chi-Square test.

Results: Data on 270 infants with median gestation 26(23-34.43) weeks and birthweight 788(435-1000) grams were collated. $91.5 \%$ received antenatal steroids. Median days ventilated and on CPAP were $1(0-99)$ and $15(0-81)$ respectively. 16 of 270(5.9\%) infants received treatment for PDA at our unit, with a further $10(3.7 \%)$ treated subsequently following discharge.

\begin{tabular}{|c|c|c|c|c|c|c|}
\hline Outcomes & $\begin{array}{c}\text { PDA } \\
\text { Treatment }\end{array}$ & $\begin{array}{r}\text { CLD at } 36 \\
\text { weeks CGA }\end{array}$ & $\begin{array}{l}\text { Severe ROP } \\
\text { (Grade III-V) }\end{array}$ & $\begin{array}{c}\text { Grade } 3 \text { or } 4 \\
\text { IVH }\end{array}$ & NEC & Mortality \\
\hline $\begin{array}{c}\text { QCCH } \\
(2007-2009)\end{array}$ & $26 / 270(9.6 \%)$ & \begin{tabular}{|l|}
$78 / 270$ \\
$(28.9 \%)$
\end{tabular} & $26 / 270(9.6 \%)$ & \begin{tabular}{|l|}
$40 / 270$ \\
$(14.8 \%)$
\end{tabular} & \begin{tabular}{|l|}
$33 / 270$ \\
$(12.2 \%)$
\end{tabular} & $81 / 270(30 \%)$ \\
\hline $\begin{array}{l}\text { VON Results } \\
\text { (2004) }\end{array}$ & $\begin{array}{l}8660 / 16557 \\
(52.3 \%)\end{array}$ & \begin{tabular}{|l|}
$6712 / 16557$ \\
$(58.7 \%)$
\end{tabular} & \begin{tabular}{|l|}
$2356 / 16557$ \\
$(14.2 \%)$
\end{tabular} & \begin{tabular}{|l|}
$2914 / 16557$ \\
$(17.6 \%)$
\end{tabular} & \begin{tabular}{|l|}
$1476 / 16557$ \\
$(8.9 \%)$
\end{tabular} & \begin{tabular}{|l}
$4608 / 16557$ \\
$(27.8 \%)$
\end{tabular} \\
\hline P Value & 0.0001 & 0.0001 & 0.039 & 0.27 & 0.075 & 0.47 \\
\hline
\end{tabular}

[Short term outcomes against VON]

Conclusion: While these data have not been risk-stratified, treatment of PDA in ELBW babies in our unit appears to be considerable lower than in $\mathrm{VON}$, but this is not associated with significant differences in outcome. A trend to increased rate of NEC requires further study. 